\title{
Formulation of Folate-conjugated, Doxorubicin- loaded Human Serum Albumin Nanoparticles for Promotion of Gene Expression Associated with Apoptosis in Renal Cell Carcinoma
}

Khalil A. A. Khalil

University of Bisha

Sharafaldin Al-Musawi ( $\nabla$ dr.sharaf@biotech.uoqasim.edu.iq )

Al-Qasim Green University

Salim Albukhaty

University of Misan

Ghassan M. Sulaiman

University of Technology

Hassan Al-Karagoly

University of Al-Qadisiyah

Elsadig M. Ahmed

University of Bisha

Mohamed T. A. Solaiman

University of Bisha

\section{Research Article}

Keywords: Folate receptor, Apoptosis, Doxorubicin, Human Serum Albumin, Nanoparticle, Cancer targeting

Posted Date: February 25th, 2021

DOl: https://doi.org/10.21203/rs.3.rs-229350/v1

License: (9) This work is licensed under a Creative Commons Attribution 4.0 International License. Read Full License 


\section{Abstract}

In this study, we developed a new, efficient targeting and pH-responsive formulation using folate (FA)conjugated human serum albumin (HSA) nanoparticles to deliver doxorubicin (DOX) drug to renal cell carcinoma. FA was conjugated to HSA, and DOX was loaded into obtained FA-HSA NP using the desolvation method. FA-HSA-DOX NPs were examined using zeta potential and (DLS) measurements, dynamic light scattering, scanning electron microscopy, atomic force microscopy, FTIR, and UV-Vis Spectroscopy. Drug liberation rate, toxicity/viability rate, and the apoptosis/necrosis effect of different DOX, FA-HSA, and FA-DOX-HSA treatments were evaluated using release profile study, MTT assay, and flow cytometry methods, respectively. The expression of BAK, BCL2, BAX, and BCL-XL genes in cancer cells after treatment was analyzed via real-time polymerase chain reaction (PCR). The average size, zeta potential, and polydispersity of FA-DOX-HSA NPs were $200 \pm 9(\mathrm{~nm}),-47 \pm 7(\mathrm{mv})$, and 0.012 , respectively. This formulation showed $\mathrm{pH}$-dependent drug release, high drug loading performance, sustained ability of drug release. The notable toxic properties and high apoptosis-stimulation ability of the FA-HAS-DOX NPS in RCC-GH cell lines demonstrated long-term anticancer efficacy. Also, real-time PCR results showed an increase in BAX expression and a decrease in BCL-2 L1 and BCL-XL significantly. Our findings indicate that FA-HSA NPs have no cytotoxic effect on cancer cells (RCC-GH) and healthy cells (RPTEC/TERT1). Our study provides a simple and efficient FA-HSA carrier with intrinsic biocompatibility for DOX drug delivery

\section{Introduction}

DOX is an efficient anticancer drug that belongs to the anthracycline antibiotics and is commonly used alone or in combination with other chemotherapy agents in the treatment of a broad range of cancers such as lymphoma, leukemia, and also breast, bladder, and kidney cancers [1-6]. However, the inability to select target cells, the harmful adverse effects such as cardiotoxicity, and the emergence of drug resistance remain significant limitations associated with DOX administration [7, 8]. Drug delivery-based nanoparticles (NPs) (especially natural polymeric NPs (have gained great attention because of their nanoscale properties and their enhanced permeability and retention of anticancer therapeutics [9-12]. High biocompatibility of natural materials such as protein-based NPs can be used as ideal drug carriers because of their distinctive characterizations such as amphiphilic, shelf life, the possibility of surface modification, and biodegradability [13]. HSA is the largest constituent of plasma proteins that contributed to the upkeep of osmotic pressure. It acts as a biocompatible, non-toxic, and biodegradable natural polymeric material. This molecule comprised amino acids connected to peptide bonds that make it possible for embedding and drug loading. HSA enhances drug stability and permits it to release into cancer cells through cellular uptake, which makes it an ideal platform to fabricate NPs for drug delivery systems $[14,15]$. The folate receptor is known as a tumor cell biomarker because it can overexpress on the surfaces of various cancer cells, encompass renal, lung, colon, brain, uterine, cervix, and breast [16]. However, it is rare to find this protein on the surface of healthy cells [17]; this difference can be used to target malignant cells to improve drug delivery. In this work, to improve targeted treatment to deliver DOX 
drug in human clear epithelial carcinoma (RCC-GH) cells in vitro model, we have focused on fabricated folic acid-coated human serum albumin NPs (HSA-FA). The prepared FA-HSA-DOX NPs were evaluated for DOX drug release, and their anticancer activity on both Human Kidney clear cell carcinoma (RCC-GH) and human renal proximal tubule (RPTEC/TERT1) cells were examined. We have also investigated the cellular proliferative changes and mRNA gene expression of pro-apoptotic gene BAX and anti-apoptotic gene BCL2 after the treatment of RCC-GH cancer cells with FA-HSA NPs that comprise DOX.

\section{Materials And Methods}

\subsection{Materials}

Dulbecco's Modified Eagle Medium (DMEM) cell culture medium, fetal bovine serum (FBS), and penicillin/streptomycin were obtained from Gibco, Life Technologies (Paisley, United Kingdom). Highpurity HSA, folate, doxorubicin hydrochloride, and MTT were obtained from Sigma-Aldrich (St. Louis, MO, USA).

\subsection{Preparation of folate-DOX-loaded HAS (FA-DOX-HAS) NPS}

FA-functionalized HSA NPs were prepared at ambient conditions using an ethanol desolvation method [18]. Briefly, $50 \mathrm{mg}$ of HSA was added to $5 \mathrm{~mL}$ of $10 \mathrm{mM} \mathrm{NaCl}$ solution under $800 \mathrm{rpm}$ stirring at room temperature. The solution was stirred continuously for $15 \mathrm{~min}$ and then its $\mathrm{pH}$ was titrated to 8.5 using $1 \mathrm{~N} \mathrm{NaOH}$ for 5 min stirring. The ethanol was added dropwise to a desolvating factor of HSA solution under continuous stirring until the HSA solution became turbid ( 1-2 mL). To form stable HSA NPs $10 \%$ glutaraldehyde (GA) as a cross-linking agent was added to the obtained turbid HSA solution. The centrifugation of this solution was performed at $14000 \mathrm{rpm}$ for $15 \mathrm{~min}$. Afterward, the NPs sedimentation was rinsed with distilled deionized water (DDW). Then, the rinsed NPs were suspended in an equal volume of PBS. FA dissolved in DDW (followed by $\mathrm{NaOH}$ addition) was added to the resulted NPs solution. The mixed solution was gently vortexed for $1 \mathrm{~min}$ to allow the electrostatic binding of FA on the surface of HSA NPs. For the nanoformulation of drug-loaded FA-HSA NPs, DOX was added to $1 \mathrm{~mL} F A$ HSA solution and kept in constant stirring for $5 \mathrm{~h}$, followed by dropwise ethanol addition.

\subsection{Characterization}

A DLS (Nanopartica SZ-100; HORIBA Ltd, Kyoto, Japan) was used to measure the polydispersity, size, and surface charge of NPs. Particle size distribution, zeta potential, and polydispersity index of NPs were measured using deionized water $(1 \mathrm{mg} / 1 \mathrm{~mL})$ through a Zeta sizer (Malvern Instruments Ltd., Malvern, UK). The morphological features were examined using SEM (Philips XL30) and AFM (Nanoscopy Digital) Instruments.

\subsection{Fourier-transform infrared spectroscopy (FTIR)}

FTIR is an effective technique for evaluating the structure and formation of NPs. A spectrometer (Nicolet, Madison, WI, USA) was applied to record the FTIR spectra from vacuum-dried samples. The FTIR 
resolution was set at $4 \mathrm{~cm}^{-1}$ and used to fit the $400-4000 / \mathrm{cm}$ spectrum scope.

\subsection{Ultraviolet-visible (UV-vis) spectrophotometry}

The appropriate combination of drugs and the successful combination of target agents plays a key role in building nanocomposites. UV-vis experiments were performed to confirm the conjugation of FA, DOX, and HSA molecules on FA-DOX-HSA NPs. The absorption spectrum of FA-DOX-HAS NPs was measured using a UV-vis spectrophotometer (Shimadzu, Tokyo, Japan) with a $1 \mathrm{~cm}$ quartz cuvette to detect absorption range at different intervals between 200 and $700 \mathrm{~nm}$.

\subsection{Encapsulation efficiency}

The NP solution was centrifuged for $20 \mathrm{~min}$ at $14000 \mathrm{rpm}$. Drug-loaded NPs (FA-DOX-HSA) were estimated, and the free unloaded DOX drug in the supernatant was measured using a UV spectrophotometer. The following equation was applied to measure encapsulation efficiency:

Encapsulation efficiency $(\%)=[($ Total drug - Free unloaded drug $) /$ Total drug $]$ Eq. (1)

\subsection{In vitro determination of DOX drug release}

To measure the DOX drug release from the prepared nanosystem FA-DOX-HSA, under in vitro conditions, membrane-drug diffusion studies were utilized. Briefly, $1 \mathrm{~mL}$ of FA-DOX-HSA suspension was transferred in a dialysis bag and then immersed in $100 \mathrm{~mL}$ phosphate buffer $(\mathrm{pH}=7.4)$ and citrate buffer $(\mathrm{pH}=5.4)$ and incubated at $37^{\circ} \mathrm{C}$ within bath shaking. One milliliter of FA-DOX-HSA suspension was drawn out, freeze-dried, and dissolved in $2 \mathrm{~mL}$ of methanol. Fluorescence spectroscopy (Cary 100 BIO UV-vis spectrophotometer, Varian, CA, USA) at $450 \mathrm{~nm}$ was used to determine the DOX amount release from FADOX-HSA formulation.

The following equation was used to calculate the DOX drug release:

$R=\left[V \otimes^{n-i} C_{i}+V_{0} C_{n}\right] / m_{\text {drug }}$ Eq. (2)

where $\mathrm{R}$ is the final drug release (\%), $\mathrm{V}$ is the volume of each sample, $\mathrm{V}_{0}$ is the initial volume of drug, $\mathrm{Ci}$ and $\mathrm{Cn}$ are the DOX concentrations, $\mathrm{i}$ and $\mathrm{n}$ are the sampling times, and $\mathrm{m}_{\mathrm{drug}}$ is the mass of DOX in NPs.

\subsection{Cell culture}

RCC-GH and RPTEC/TERT1 cell lines were purchased from ATCC (UK). All cells were plated and cultured in DMEM enriched with $10 \% \mathrm{FBS}$ and glutamine $(2 \mathrm{mM})$ and incubated in $37^{\circ} \mathrm{C}$ and $5 \% \mathrm{CO}_{2}$ atmosphere.

\subsection{Cellular internalization}

The fluorescence emission spectrum of DOX was inspected in various biological medium to evaluate the ability and activity of the FA-DOX-HSA formulation in DOX protection. For this purpose, the cells were 
treated with $25 \mu \mathrm{g} \mathrm{mL}{ }^{-1}$ FA-DOX-HSA. Additionally, FA-HSA and DOX with the same concentration served as references. The imaging process was performed using a fluorescence microscope (Nikon Eclipse TE2000-U) in the range of 420 to $70 \mathrm{~nm}$.

\subsection{MTT assay}

RCC-GH and RPTEC/TERT1 cells (in cultured media) were seeded into 96-well plates at a density of $1 \times 10^{5}$ cells per well and incubated overnight to make the cells adhere to the wells. Then, the media were changed with achieved concentrations of FA-HSA, DOX, and FA-DOX-HSA NPs that were dissolved in a medium with a range of 10 to $60 \mu \mathrm{M}$. Following 24 and $48 \mathrm{~h}$ of treatment. The toxicity was examined after adding a $5 \mathrm{mg} / \mathrm{mL}$ MTT solution (a further $4 \mathrm{~h}$ incubation). Then, the media were removed and $100 \mu \mathrm{L}$ of DMSO was added to each well. The absorbance was measured at $570 \mathrm{~nm}$, on $690 \mathrm{~nm}$ wavelength using a microplate reader (Tecan Group Ltd., Männedorf, Switzerland).

\subsection{Flow cytometry}

Cell apoptosis was conducted using flow cytometry analysis based on Annexin V-FITC Apoptosis Detection Kit (Biovision, Inc.) $1.0 \times 10^{5}$ cells per well were seeded into six-well plates, and after 1 day of plating, the cells were treated with FA-DOX-HSA, void DOX, and FA-HSA NPs and incubated for $48 \mathrm{~h}$. PBS was used to wash the cells twice and then trypsinized, and then, the cells were suspended in Annexin binding buffer using Annexin V-FITC and propidium iodide stain.

\subsection{Apoptosis assay by quantitative real-time PCR}

After treating the RCC-GH cells with DOX, FA-HSA, and DOX-loaded FA-HSA NPs, they were transferred to a six-well plate at a density of $1 \times 10^{5}$ cells $/ \mathrm{mL}$ and cultured in a humidified atmosphere for $48 \mathrm{~h}$. RNA was extracted from cells after $48 \mathrm{~h}$ of treatment by using TRIzol according to the manufacturers' protocol (Invitrogen Life Technologies, UK). Complementary DNA (cDNA) synthesis was achieved based on the protocol of the RevertAid M First-Strand cDNA Synthesis Kit. The reaction was conducted in iCycler thermal cycler (Bio-Rad, Hercules, CA, USA) following the protocol cycle that involved $25^{\circ} \mathrm{C}$ for 6 min, incubation at $42^{\circ} \mathrm{C}$ once more for $1 \mathrm{~h}$, and then heating at $75^{\circ} \mathrm{C}$ for 5 min. CDNA is directly used in the real-time PCR. Table 1 mentions the primers for targets and endogenous genes, designed using software primer express. Amplification reactions contained $5 \mu \mathrm{L}$ of cDNA, $10 \mu \mathrm{L}$ of the SYBR Green-I dye (Applied Biosystems, USA), and $0.5 \mu \mathrm{L}$ of each of the specific primers. Primers' concentration in the final volume of $20 \mu \mathrm{L}$ was $100 \mathrm{nM}$. Real-time PCR was performed as follows: 50 cycles initiated at $95^{\circ} \mathrm{C}$ for $10 \mathrm{~min}$ and then at $95^{\circ} \mathrm{C}$ for $15 \mathrm{~s}$ and $60^{\circ} \mathrm{C}$ for $1 \mathrm{~min}$; the real-time PCR success was evaluated with the melting curve analysis.

\subsection{Statistical analysis}

Statistical analysis was conducted using SPSS software (version 22), and data were expressed as unpaired Student's t-test, standard error of the mean (mean \pm SEM), and analysis of variance (ANOVA) 
with ${ }^{*} p<0.05$ considered as statistically significant.

\section{Results And Discussion}

\subsection{Characterization of FA-HAS-DOX NPs}

Recently, various researches have focused on HSA as a promising agent for carrying anticancer drugs. Although the utilization of organic solvents to prepare NPs is preferred, many materials are difficult to dissolve in both water and organic solvent. One way to prepare such NPs without applying organic solvents is to use ethanol [23].

In the present study, the ethanol desolation method was used to produce FA-HAS-DOX NPs, in which ethanol was used as a desolvating factor and GA as a cross-linking media. GA is a water-soluble, lowcost, and bifunctional substance with notable reactivity. The cross-linking process among protein structures happened by the two carbonyl groups of GA and due to the nucleophilic attack of the $\varepsilon$-amino groups in lysine and arginine residues. The NPs size will not change due to GA reaction process but the surface charge is significantly affected. $[24,25]$. Thus, GA was used as the cross-linking, stabilizing agent in aqueous and cellular media.

The dispersion of HSA in water was performed, and ethanol was used as the dehydrating agent after $\mathrm{pH}$ was adjusted to alkaline value. This method is regarded as a simple and low-cost technique and produces a lower aggregation rate with uniform NP distribution than those in other methods [26]. According to a study conducted by Lomis et al., the particle size might be moderately affected with an alteration of $\mathrm{pH}$ in the range of approximately $100-200 \mathrm{~nm}$ [27]. DLS and Zeta data of synthesized FADOX-HSA exhibited that the sample provided an overall diameter size of $200 \pm 9 \mathrm{~nm}$, which is consistent with previous studies [28]. The polydispersity was 0.012 , which presents a negligible diversity of the NP size and a $-47.2 \mathrm{mV}$ charge (Fig. 1A and B), which shows high stability and is desirable for different biomedical applications [29]. Besides, Li Q et al. indicated that the particle size of hydroxy-camptothecin encapsulated by HSA and modified by FA was $233.9 \pm 1.2 \mathrm{~nm}$ and the zeta charge was $-25.23 \pm 2.98 \mathrm{mV}$ [25]. The results provided by SEM together with AFM agreed with the results obtained with Zeta analysis, indicated that FA-DOX-BSA NPs have discrete spherical shapes that can be detached from their neighbor with homogeneous surface density (Fig. 2A and B). The loading activity of FA-DOX-HAS NPs was 79\%.

\subsection{FTIR spectroscopy}

Figure 3 reveals the FTIR spectra of FA, HSA, FA-HSA NPs, DOX, and FA-HSA-DOX NPs. Figure 3 (i) shows an infrared spectrum peak of bare FA (the stretching vibration of the benzene ring skeleton at $1500 / \mathrm{cm}^{-}$ ${ }^{1}$ ). The second peak is located at $1602 \mathrm{~cm}^{-1}$, which corresponded to the spectrum of the bare DOX, as shown in Fig. 3 (ii). In Fig. 3 (iii), the HSA absorption peak was $1790 \mathrm{~cm}^{-1}$, due to a large number of amino acids compound in this molecule. The infrared spectrum of FA-HSA NPs as shown in Fig. 3 ( $(\mathbb{)})$ contains the characteristic peaks of FA and has a peak at $3410 \mathrm{~cm}^{-1}$. The infrared spectrum of the FADOX-HSA nanoformulation has an absorption peak at $1651 \mathrm{~cm}^{-1}$ and $3324 \mathrm{~cm}^{-1}$. A small peak appeared 
in the infrared spectrum of FA-HSA-DOX NPs (Fig. 3(v)), indicating the presence of a certain amount of DOX on the surface of FA-HSA NPS.

\subsection{UV-vis spectroscopy}

UV-vis spectroscopy is used to further test the encapsulation of FA, HSA, and DOX in the NPs. Results indicated the spectral data details of the energy band gaps and optical transition to prove the anticancer drug bonding to the FA-HSA. The UV-vis spectra of pure FA, HSA, and Dox and their NP counterparts were significantly different. The spectra exhibited an absorbance peak at 550, 340, and $310 \mathrm{~nm}$, respectively, whereas both a mixture of free drugs and FA-DOX-HSA NPs exhibited a broad absorbance peak at 510 nm (Fig. 4).

\subsection{Release profile}

Figure 5 shows that the DOX drug liberation from FA-DOX-HSA was achieved in the presence of PBS (0.01 $\mathrm{M}, \mathrm{pH}=7.4)$ and citrate buffer $(0.01 \mathrm{M}, \mathrm{pH}=5.4)$ at $37^{\circ} \mathrm{C}$. The amount of released DOX from FA-DOX-HSA NPs was examined by counting fluorescence intensity emission from the supernatant at changed $\mathrm{pH}$ values. The drug liberation data indicated that the release time from the FA-DOX-HAS nanocarriers at a 96 $\mathrm{h}$ time was slower at $\mathrm{pH} 7.4$ than at $\mathrm{pH}$ 5.4. Additionally, the free DOX release ratio showed a similar release behavior in both $\mathrm{pH} 7.4$ and 5.4. The release curve analysis exhibited a faster liberation profile for DOX at $\mathrm{pH} 5.4$ than at $\mathrm{pH} 7.4$ [30].

\subsection{Cellular internalization}

Figure 6 shows the experimental results of cell internalization. The cell uptake assay was performed using fluorescence microscopy that presented green fluorescent expression in the RCC-GH-cells cytoplasm after subjected to FA-DOX-HSA NPs [31].

\subsection{MTT assay}

Results of cytotoxic effects of the FA-DOX-HSA NPs were checked by MTT assay against RCC-GH and RPTEC/TERT1 cell lines, as revealed in Fig. 7. The cancer cells were treated with both void DOX and bare FA-DOX-HSA even at the highest concentration $60 \mu \mathrm{M} \mathrm{mL}^{-1}$ showed no toxicity. Approximately more than $80 \%$ of cells remain alive after $48 \mathrm{~h}$ of treatment, which exhibited the cytocompatibility of both void DOX and bare FA-DOX-HSA treatments. Cell toxicity increased noticeably when subjected to FA-DOX-HSA NPs and showed a remarkable cell growth prevention ratio of RCC-GH cells as compared with void DOX and free FA-HSA NPs. The IC50 value was calculated by a concentration-ratio curve fitting of the cell toxicity results. The IC50 values of FA-DOX-HSA NPs for RCC-GH cells within 24 and $48 \mathrm{~h}$ were 48 and $27 \mu \mathrm{M}$, respectively. This observation might be explained by the observation of more apoptotic as opposed to necrotic cells after treatment with the FA-DOX-HSA NPs. However, it is necessary to elucidate the processes of the anti-proliferative inhibitory properties of FA-DOX-HSA NPs for the standardization and development of an efficient treatment regime.

\subsection{Flow Cytometry}


Figure 8 shows the cell apoptosis analysis. The results exhibited that the treatment of RCC-GH cells with bare nano-carrier or void DOX drug did not present noticeable apoptosis induction after $24 \mathrm{~h}$ of treatment. Conversely, the level of RCC-GH cells affected by apoptosis was increased remarkably when subjected to FA-DOX-HSA nanoformulation. These findings confirm that FA-DOX-HSA has notable potential to kill RCC$\mathrm{GH}$ cells using DOX and noticeably induce programmed cell death in cancer cells. Moreover, it was not possible to make a distinction between these two patterns of cell death only by using the progressive loss in the integrity of the plasma membrane as an indicator [32]. The use of NPs as capsules for anticancer drugs can facilitate their intake by the cells and the lysosomes, leading to more highly induced cytotoxic activity [33-36]. One previous investigation indicated that increasing the development of intracellular ROS could be responsible, at least partially, for the killing effect of anticancer drugs [32]. The incubation of cancer cells with NPs has been associated with altered cell proliferation and induced apoptosis, influenced by changes in ROS development levels [19]. However, events such as increased morphological apoptotic alterations and DNA damage may be due to elevated ROS concentrations and associated mitochondrial membrane alterations.

\subsection{Real-time PCR and gene expression profile}

Bcl-2 family proteins can activate Bax, Bak (pro-apoptotic) and Bcl-2, Bcl-xl (anti-apoptotic) cells inhibition. Researchers have indicated that the ratio changes to pro-apoptotic and anti-apoptotic proteins regulated cellular apoptosis [20,21,37]. N Pilco-Ferreto reported the requirements of BCL2 expression in response to DOX [38]. We have investigated the expression of the mentioned genes in RCC-GH after treatment with FA-DOX-HSA NPs in comparison with the FA-HSA and void DOX, in which after treatment, the FA-DOX-HSA significantly decreased the expression of Bcl-2 and Bcl-xl of the control group (untreated). Moreover, FA-DOX-HSA considerably upregulated the expression of BAK and Bax genes of healthy levels $(P<0.01)$ (Fig. 9).

\section{Conclusions}

The results of the study concluded that the FA-DOX-HSA nanoparticles were successfully developed and demonstrated the effective cellular uptake of DOX anti-cancer drug, on the other hands The conjugates between FA-HSA nanoparticles and DOX drug are of good high activity with pH-dependent drug release, these conjugates act as synergistic biocompatible treatment, with low cellular toxicity effect when compared to DOX drug alone, that could be used for future cancer therapy.

\section{Declarations}

\section{Conflicts of Interest:}

The authors declare no conflict of interest.

\section{References}


[1] N. Zhao, M.C. Woodle, A.J. Mixson, Advances in delivery systems for doxorubicin. J. Nanomed. Nanotechnol. 9(5), (2018).

[2] Y. Zhang, Y. He, L.L. Lu, Z.Y. Zhou, N.B. Wan, G.P. Li, X. He, H.W. Deng, miRNA-192-5p impacts the sensitivity of breast cancer cells to doxorubicin via targeting peptidylprolyl isomerase A. Kaohsiung J. Med. Sci. 35(1), 17-23 (2019).

[3] W. Fukuokaya, T. Kimura, J. Miki, S. Kimura, H. Watanabe, F. Bo, D. Okada, K. Aikawa, A. Ochi, K. Suzuki, Effectiveness of Intravesical Doxorubicin Immediately Following Resection of Primary Nonmuscle-invasive Bladder Cancer: A Propensity Score-matched Analysis. Clin. Genitourin. Cance.r 18(2), e55-e61 (2020)

[4] T. Etrych, L. Daumová, E. Pokorná, D. Tušková, O. Lidický, V. Kolářová, J. Pankrác, L. Šefc, P. Chytil, P. Klener, Effective doxorubicin-based nano-therapeutics for simultaneous malignant lymphoma treatment and lymphoma growth imaging. J. Control. Release. 289, 44-55 (2018)

[5] B. Zhu, H. Zhang, L. Yu, Novel transferrin modified and doxorubicin loaded Pluronic 85/lipid-polymeric nanoparticles for the treatment of leukemia: in vitro and in vivo therapeutic effect evaluation. Biomed Pharmacother. 86, 547-554 (2017)

[6] S. Zhao, H. Yu, N. Du, Experimental study of doxorubicin interventional chemotherapy in the treatment of rabbit VX2 renal transplantation carcinoma. Int. J. Clin. Exp. Med. 8(7) (2015) 10739.

[7] Y.L. Franco, T.R. Vaidya, S. Ait-Oudhia, Anticancer and cardio-protective effects of liposomal doxorubicin in the treatment of breast cancer. Breast Cancer: Targets and Therapy. 10, 131 (2018)

[8] M.A. Al-Kinani, A.J. Haider, S. Al-Musawi, High Uniformity Distribution of Fe@ Au Preparation by a Micro-Emulsion Method. IOP Conference Series: Materials Science and Engineering, IOP Publishing, 2020, pp. 012013.

[9] S. Al-Musawi, M.J. Kadhim, N.K.K. Hindi, Folated-nanocarrier for paclitaxel drug delivery in leukemia cancer therapy. J. Pharm. Sci. Res. 10(4), 749-754 (2018)

[10] M.J. Al-Awady, A.A. Balakit, S. Al-Musawi, M.J. Alsultani, A. Kamil, M. Alabbasi, Investigation of AntiMRSA and Anticancer Activity of Eco-Friendly Synthesized Silver Nanoparticles from Palm Dates Extract. Nano. Biomed.Eng. 11(2), (2019).

[11] S. Al-Musawi, S. Albukhaty, H. Al-Karagoly, G.M. Sulaiman, M.S. Alwahibi, Y.H. Dewir, D.A. Soliman, H. Rizwana, Antibacterial Activity of Honey/Chitosan Nanofibers Loaded with Capsaicin and Gold Nanoparticles for Wound Dressing. Molecules. 25(20), 4770 (2020)

[12] J.M.A. Mofazzal, S. AL Musawi, M. Pirestani, R.M. Fasihi, K. Ahmadi, H. Rajayi, H.Z. Mohammad, M. Kamali, R. Mirnejad, Curcumin-loaded chitosan tripolyphosphate nanoparticles as a safe, natural and 
effective antibiotic inhibits the infection of Staphylococcus aureus and Pseudomonas aeruginosa in vivo. Iran. J. Biotechnol.12(3), 1-8 (2014).

[13] M. Tarhini, H. Greige-Gerges, A. Elaissari, Design and Synthesis of Nanoencapsulation with a New Formulation of Fe@Au-CS-CU-FA NPs by Pulsed Laser Ablation in Liquid (PLAL) Method in Breast Cancer Therapy: In Vitro and In Vivo. Int. J. Pharm. 522(1-2), 172-197 (2017)

[14] C. Tao, Y.J. Chuah, C. Xu, D.-A. Wang, Albumin conjugates and assemblies as versatile bio-functional additives and carriers for biomedical applications. J. Mater. Chem. B. 7(3), 357-367 (2019)

[15] R. Mohammadinejad, S. Karimi, S. Plant-derived nanostructures: types and applications. Iravani, R.S. Varma, Green Chem. 18(1), 20-52 (2016)

[16] S. Al-Musawi, A.J. Hadi, S.J. Hadi, N.K.K. Hindi, Preparation and Characterization of Folated Chitosan/Magnetic Nanocarrier for 5-Fluorouracil Drug Delivery and Studying its Effect in Bladder Cancer Therapy. J. Glob. Pharm. Technol. 11, 628-637 (2009)

[17] M. Kim, S. Pyo, C.H. Kang, C.O. Lee, H.K. Lee, S.U. Choi, C.H. Park, Folate receptor 1 (FOLR1) targeted chimeric antigen receptor (CAR) T cells for the treatment of gastric cancer. PloS one. 13(6), e0198347 (2018)

[18] E. Bronze-Uhle, B. Costa, V. Ximenes, P. Lisboa-Filho, Synthetic nanoparticles of bovine serum albumin with entrapped salicylic acid. Nanotechnol. Sci. Appl. 10, 11 (2017)

[19] M.A. El Gendy, B.H. Ali, K. Michail, A.G. Siraki, A.O. El-Kadi, Induction of quinone oxidoreductase 1 enzyme by Rhazya stricta through Nrf2-dependent mechanism. J. Ethnopharmacol. 144(2), 416-424 (2012)

[20] N. Zucchini, G. de Sousa, B. Bailly-Maitre, J. Gugenheim, R. Bars, G. Lemaire, R. Rahmani, Regulation of $\mathrm{Bcl}-2$ and $\mathrm{Bcl}-\mathrm{xL}$ anti-apoptotic protein expression by nuclear receptor PXR in primary cultures of human and rat hepatocytes. Biochim. Biophys. Acta. Mol. Cel/Res. 1745(1), 48-58 (2005)

[21] B.G. Eimani, M.H. Sanati, M. Houshmand, M. Ataei, F. Akbarian, N. Shakhssalim, Expression and prognostic significance of bcl-2 and bax in the progression and clinical outcome of transitional bladder cell carcinoma. Cell Journal (Yakhteh). 15(4), 356 (2014)

[22] S. Al-Musawi, S. Albukhaty, H. Al-Karagoly, F. Almalki, Design and Synthesis of Multi-Functional Superparamagnetic Core-Gold Shell Nanoparticles Coated with Chitosan and Folate for Targeted Antitumor Therapy. Nanomaterials. 11(1), 32 (2021)

[23] B. Lian, M. Wu, Z. Feng, Y. Deng, C. Zhong, X. Zhao, Folate-conjugated human serum albuminencapsulated resveratrol nanoparticles: preparation, characterization, bioavailability and targeting of liver tumors. Artif. Cells Nanomed. Biotechnol. 47(1), 154-165 (2019) 
[24] S. Sebak, M. Mirzaei, M. Malhotra, A. Kulamarva, S. Prakash, Human serum albumin nanoparticles as an efficient noscapine drug delivery system for potential use in breast cancer: preparation and in vitro analysis. Int. J. Nanomedicine. 5, 525 (2010)

[25] Q. Li, C. Liu, X. Zhao, Y. Zu, Y. Wang, B. Zhang, D. Zhao, Q. Zhao, L. Su, Y. Gao, Preparation, characterization and targeting of micronized 10-hydroxycamptothecin-loaded folate-conjugated human serum albumin nanoparticles to cancer cells. Int. J. Nanomedicine. 6, 397 (2011)

[26] L. Ma'mani, S. Nikzad, H. Kheiri-Manjili, S. Al-Musawi, M. Saeedi, S. Askarlou, A. Foroumadi, A. Shafiee, Curcumin-loaded guanidine functionalized PEGylated I3ad mesoporous silica nanoparticles KIT6: Practical strategy for the breast cancer therapy. Eur. J. Med. Chem. 83, 646-654 (2014)

[27] N. Lomis, S. Westfall, L. Farahdel, M. Malhotra, D. Shum-Tim, S. Prakash, Human serum albumin nanoparticles for use in cancer drug delivery: process optimization and in vitro characterization. Nanomaterials. 6(6), 116 (2016)

[28] G.M. Sulaiman, Molecular structure and anti-proliferative effect of galangin in HCT-116 cells: in vitro study. Food Sci. Biotechnol. 25(1), 247-252 (2016)

[29] G.M. Sulaiman, In vitro study of molecular structure and cytotoxicity effect of luteolin in the human colon carcinoma cells. Food Sci. Biotechnol. 241(1), 83-90 (2015)

[30] S. Albukhaty, S. Al-Musawi, S. Abdul Mahdi, G.M. Sulaiman, M.S. Alwahibi, Y.H. Dewir, D.A. Soliman, H. Rizwana, Investigation of Dextran-Coated Superparamagnetic Nanoparticles for Targeted Vinblastine Controlled Release, Delivery, Apoptosis Induction, and Gene Expression in Pancreatic Cancer Cells.

Molecules. 25(20), 4721 (2020)

[31] S. Al-Musawi, S. Albukhaty, H. Al-Karagoly, G.M. Sulaiman, M.S. Jabir, H. Naderi-Manesh, Dextrancoated superparamagnetic nanoparticles modified with folate for targeted drug delivery of camptothecin. Adv. Nat. Sci-Nanosci. 11(4), 045009 (2020)

[32] A. Raza, U. Hayat, T. Rasheed, M. Bilal, H.M. Iqbal, Smart Nanoformulation Based on Polymeric Magnetic Nanoparticles and Vincristine Drug: A Novel Therapy for Apoptotic Gene Expression in Tumor. J. Mater. Res. 8(1), 1497-1509 (2019)

[33] W.J. Al-Kaabi, S. Albukhaty, A.J. Al-Fartosy, H.K. Al-Karagoly, S. Al-Musawi, G.M. Sulaiman, Y.H. Dewir, M.S. Alwahibi, D.A. Soliman, Development of Inula graveolens (L.) Plant Extract Electrospun/Polycaprolactone Nanofibers: A Novel Material for Biomedical Application. Appl. Sci. 11(2), $828(2021)$

[34] S. Albukhaty, L. Al-Bayati, H. Al-Karagoly, S. Al-Musawi, Preparation and characterization of titanium dioxide nanoparticles and in vitro investigation of their cytotoxicity and antibacterial activity against Staphylococcus aureus and Escherichia coli. Anim. Biotechnol. 1-7 (2020) 
[35] M.A. Al-Kinani, A.J. Haider, S. Al-Musawi, Design, Construction and Characterization of Intelligence Polymer Coated Core-Shell Nanocarrier for Curcumin Drug Encapsulation and Delivery in Lung Cancer Therapy Purposes. J. Inorg. Organomet. Polym. Mater. 1-10 (2020)

[36] J. Zhou, L. Zhang, M. Wang, L. Zhou, X. Feng, L. Yu, J. Lan, W. Gao, C. Zhang, Y. Bu, Design, CPX targeting DJ-1 triggers ROS-induced cell death and protective autophagy in colorectal cancer.Theranostics. 9(19), 5577 (2019)

[37] M. Jabir, U.I. Sahib, Z. Taqi, A. Taha, G. Sulaiman, S. Albukhaty, A. Al-Shammari, M. Alwahibi, D. Soliman, Y.H. Dewir, Linalool-Loaded Glutathione-Modified Gold Nanoparticles Conjugated with CALNN Peptide as Apoptosis Inducer and NF-KB Translocation Inhibitor in SKOV-3 Cell Line. Int. J.

Nanomedicine.15, 9025 (2020)

[38] N. Pilco-Ferreto, G.M. Calaf, Influence of doxorubicin on apoptosis and oxidative stress in breast cancer cell lines. Int J Oncol. 49(2), 753-762 (2016)

\section{Tables}

Table 1: The primer sequences that were utilized to amplify the genes.

\begin{tabular}{|c|c|c|c|}
\hline Gene & Forward primer sequence & Reverse primer sequence & Ref. \\
\hline$\beta$-actin & 5'-CTGGCACCCAGCACAATG-3' & 5'-GCCGATCCACACGGAGTACT-3' & [19] \\
\hline Bcl-2 & 5'- GATTGTGGCCTTCTTTGAG-3' & 5'- CAAACTGAGCAGAGTCTTC-3' & [20] \\
\hline Bcl-xl & 5'- CAGAGCTTTGAACAGGTAG-3' & 5'- GCTCTCGGGTGCTGTATTG-3' & [20] \\
\hline BAX & 5'- GGTTGTCGCCCTTTTCTA-3 & 5'- CGGAGGAAGTCCAATGTC-3' & [21] \\
\hline Bak & 5'-ACTGGGATCGAGACATGTG-3' & 5'-AGAAGGTGATGTGTACATTGC-3' & [22] \\
\hline
\end{tabular}

\section{Figures}


A

$\begin{array}{rllll} & & \text { Size (d.nm): } & \text { \% Intensity: } & \text { St Dev (d.nm): } \\ \text { Z-Average (d.nm): } 200.0 & \text { Peak 1: } & 208.9 & 100.0 & 48.08 \\ \text { Pdl: } 0.012 & \text { Peak 2: } & 0.000 & 0.0 & 0.000 \\ \text { Intercept: } 0.945 & \text { Peak 3: } & 0.000 & 0.0 & 0.000\end{array}$

Result quality : Good

\section{B}

Zeta Potential (mV): -47.2

Zeta Deviation (mV): 6.25

Conductivity (m S/cm): 0.0160

Result quality : Good

\begin{tabular}{|c|c|c|c|}
\hline & Mean (mV) & Area (\%) & St Dev (mV) \\
\hline Peak 1: & -47.2 & 100.0 & 6.25 \\
\hline Peak 2: & 0.00 & 0.0 & 0.00 \\
\hline Peak 3: & 0.00 & 0.0 & 0.00 \\
\hline
\end{tabular}

Size Distribution by Intensity

Zeta Potential Distribution
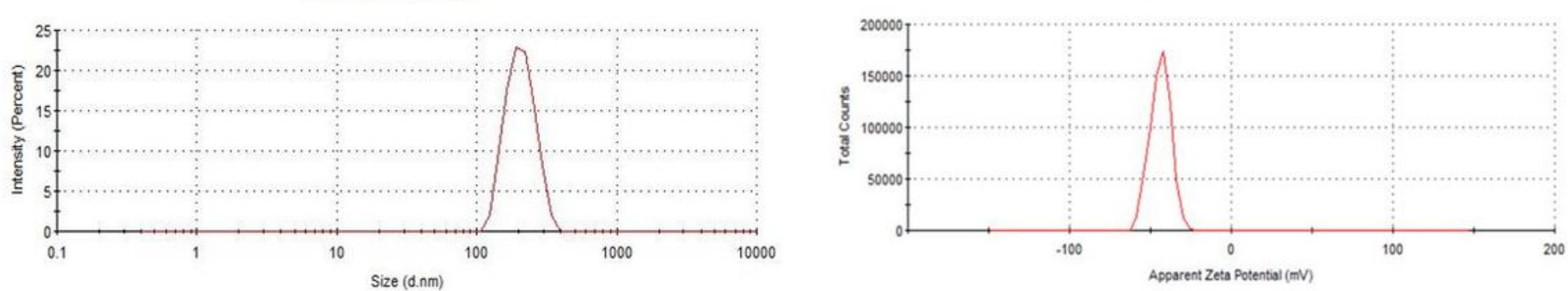

\section{Figure 1}

Measured Size, charge, and the polydispersity for stability assay of FA-DOX-HAS NPs. Z-Average or Diameter size and polydispersity of FA-DOX-HSA (A) Zeta potential of FA-DOX-HSA (B).
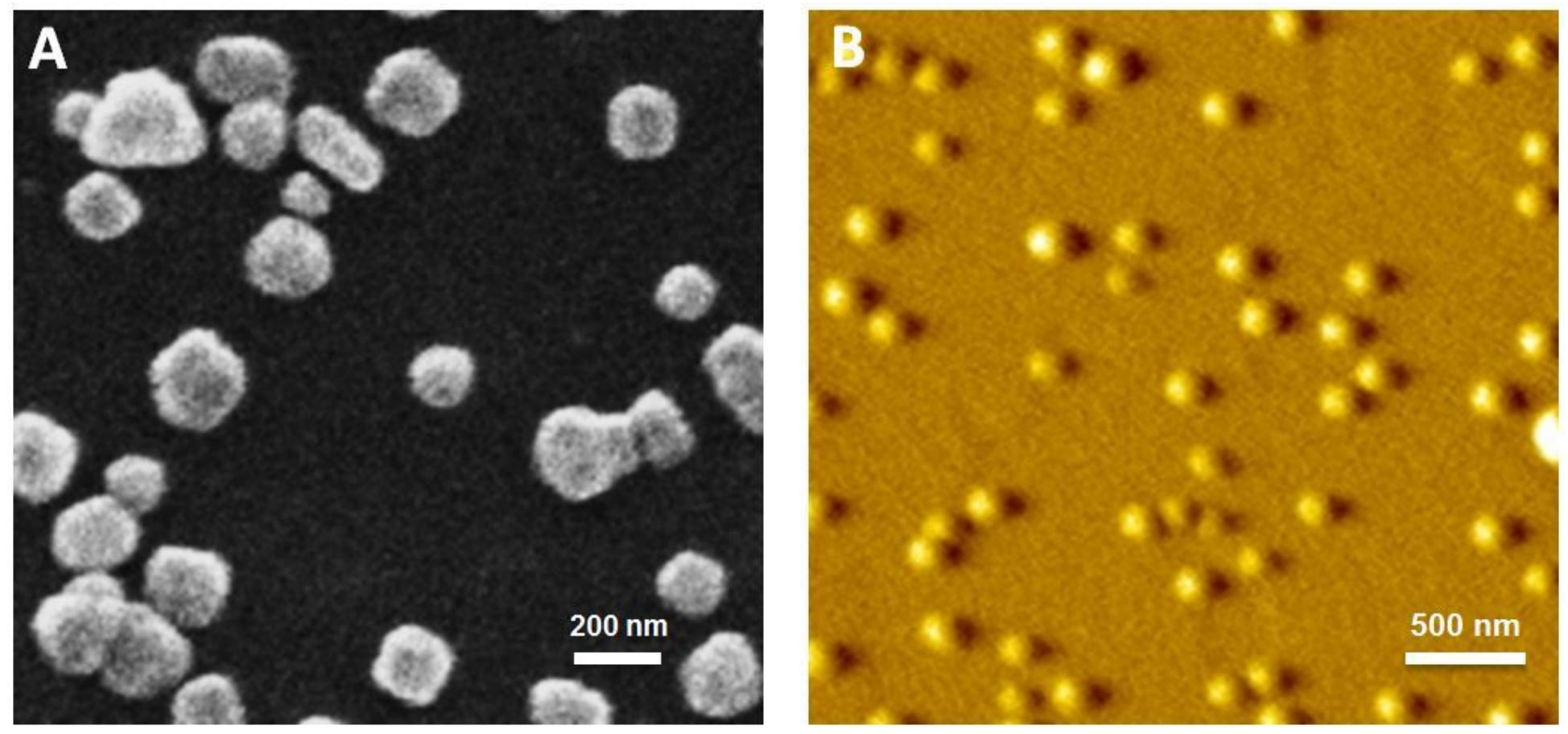

\section{Figure 2}

Microscopic analysis of FA-DOX-HSA (A) SEM image of FA-DOX-HSA (B) AFM image of FA-DOX-HSA. 


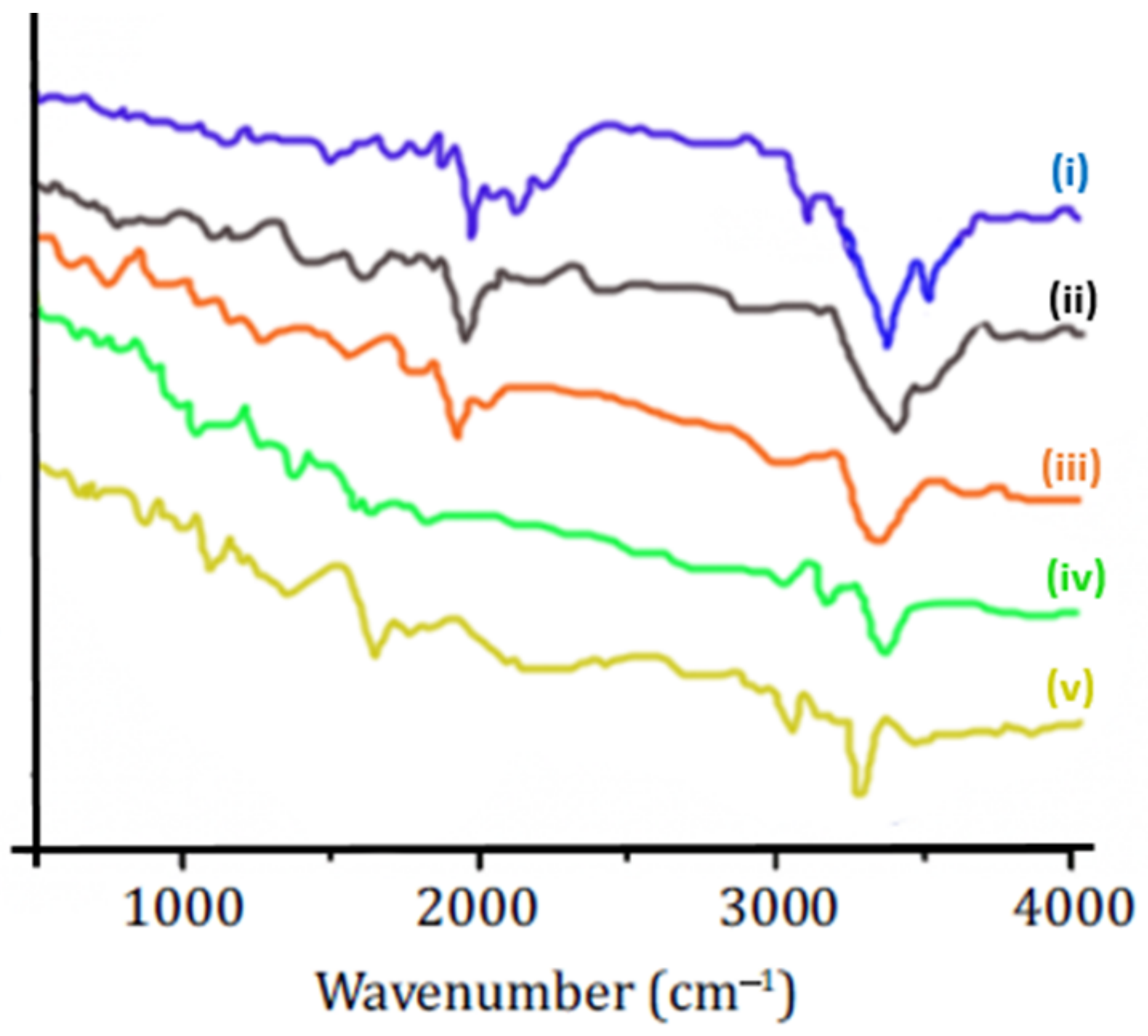

Figure 3

FT-IR spectra of FA ( (), DOX ( (), HSA ( (), FA-HSA ( $),$ FA-DOX-HSA ( () 


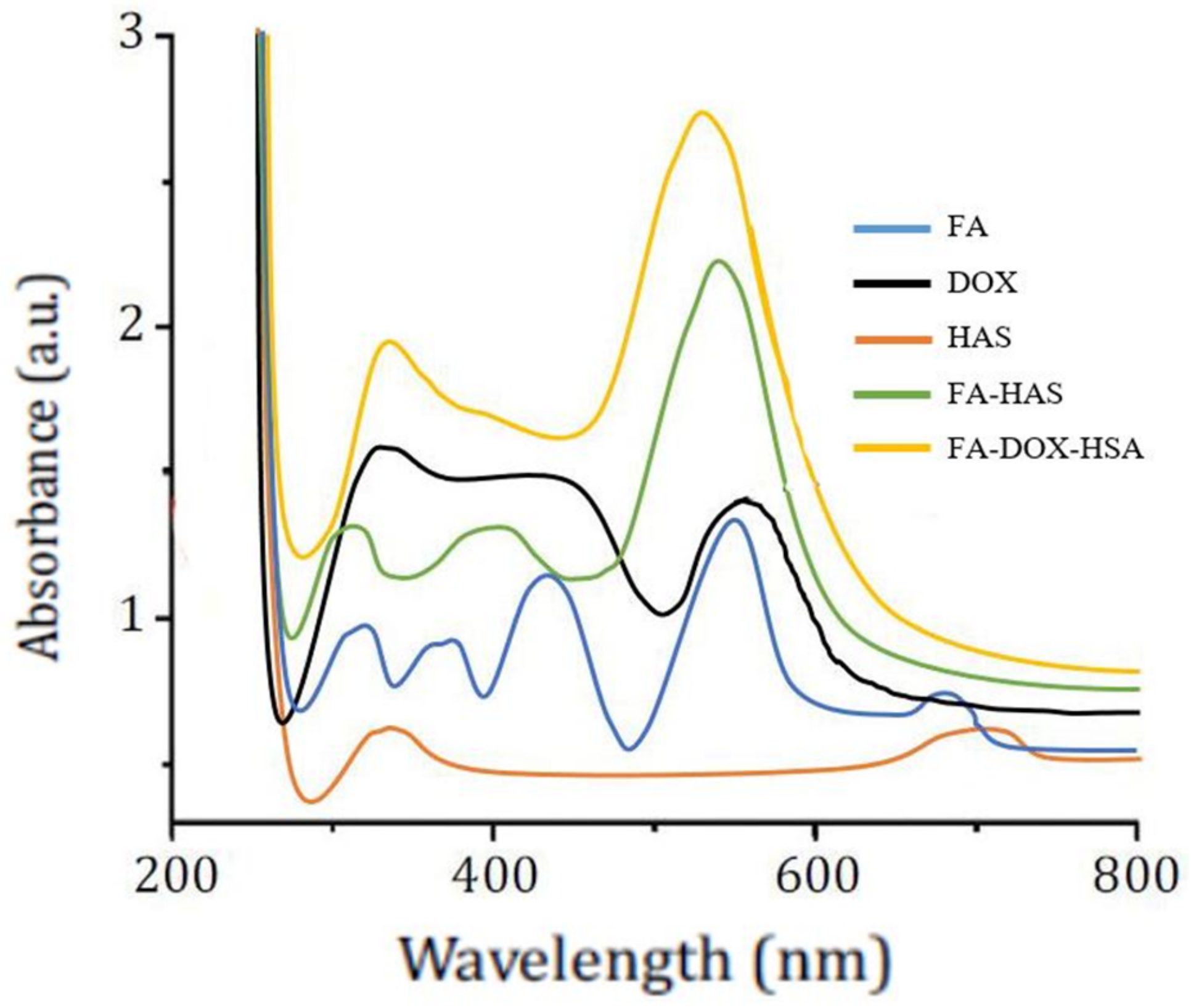

Figure 4

UV-vis spectra for FA, bar DOX, HAS, FA-HAS, and FA-HAS-DOX NPs. 


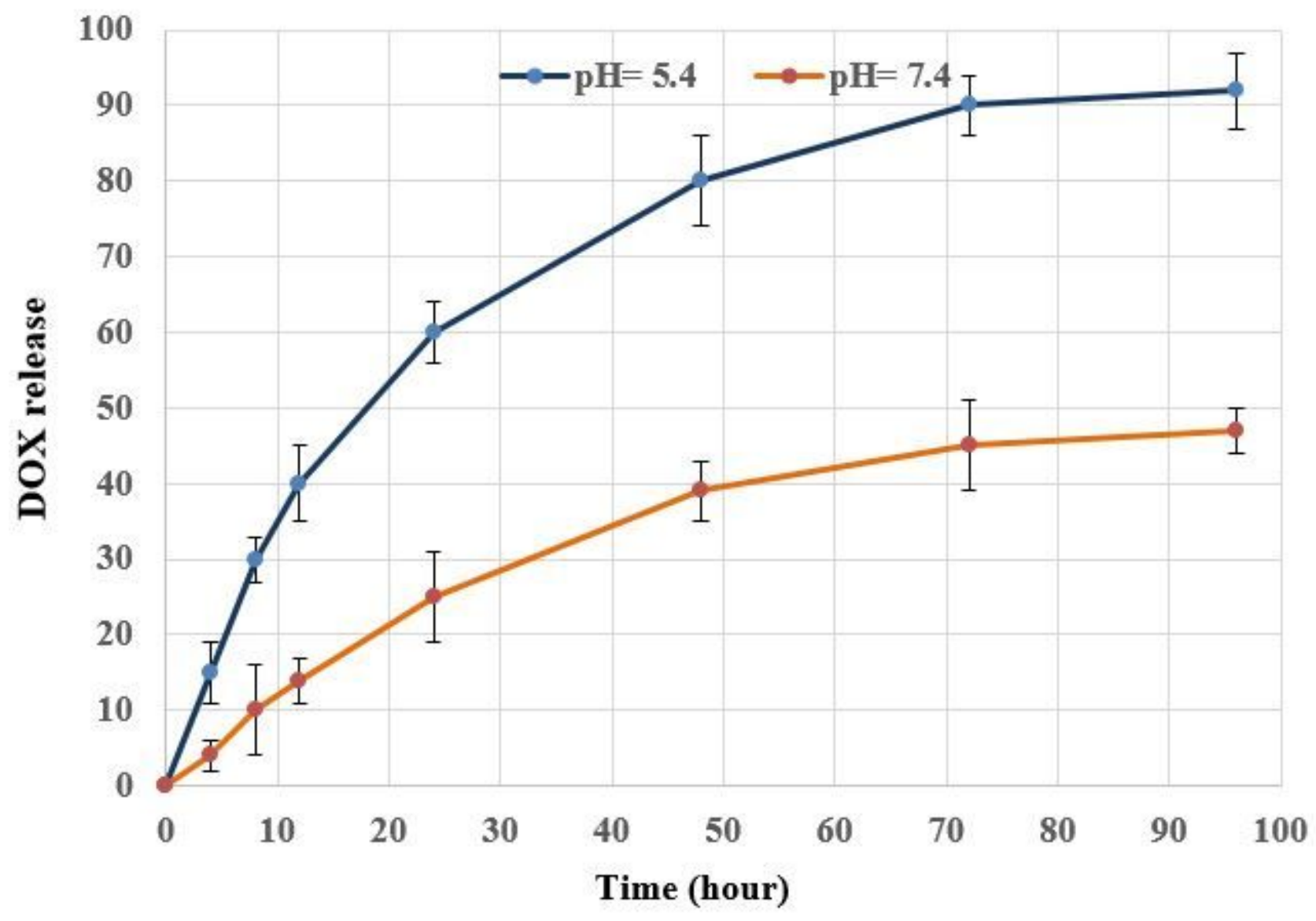

\section{Figure 5}

In vitro release profile of FA-DOX-HSA at $\mathrm{pH} 7.4$ and $\mathrm{pH}$ 5.4. All experiments were performed at $37^{\circ} \mathrm{C}$. The data represent mean values $\pm S D(n=3)$.

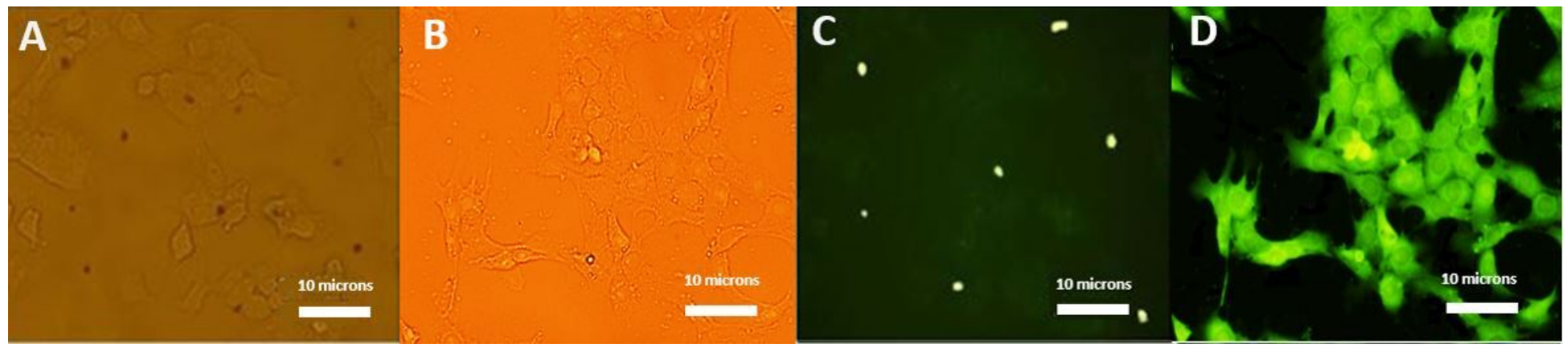

\section{Figure 6}

Cell internalization study of FA-DOX-HSA in RCC-GH cell line using a fluorescence microscope (400x magnification). Optic microscopy image of DOX treated cells (A). Optic microscopy image of FA-DOXHSA treated cells (B). Fluorescence microscopy image of DOX treated cells (C). Fluorescence microscopy image of FA-DOX-HSA treated cells (D). 

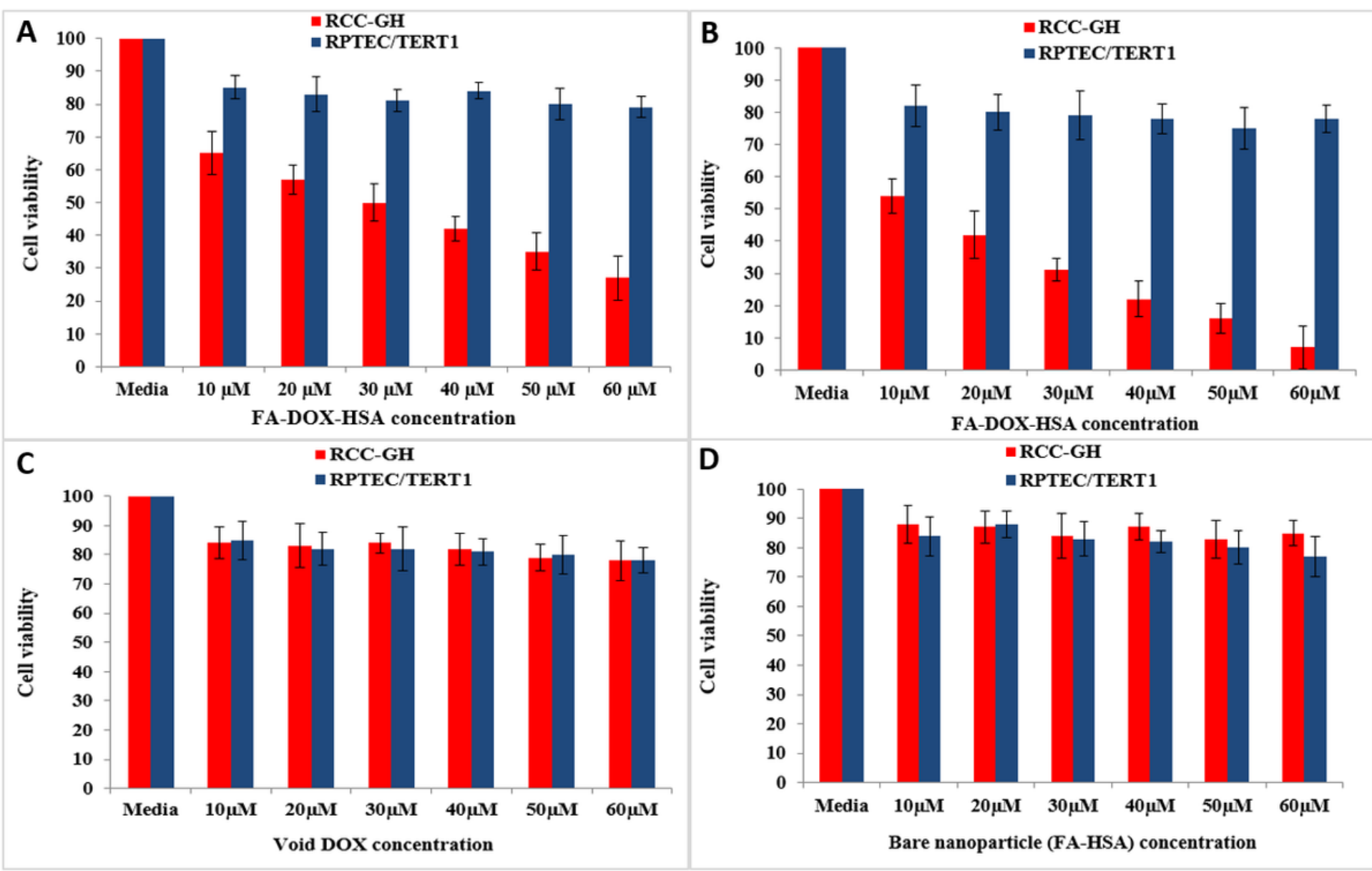

Figure 7

MTT Assay for FA-DOX-HSA NPs at $24 \mathrm{~h}(\mathrm{~A}), 48 \mathrm{~h}(\mathrm{~B})$, and void DOX (C) \& FA-HSA (D) at $48 \mathrm{~h}$ on cancer cells (RCC-GH) and healthy cells (RPTEC/TERT1).

FA-HSA

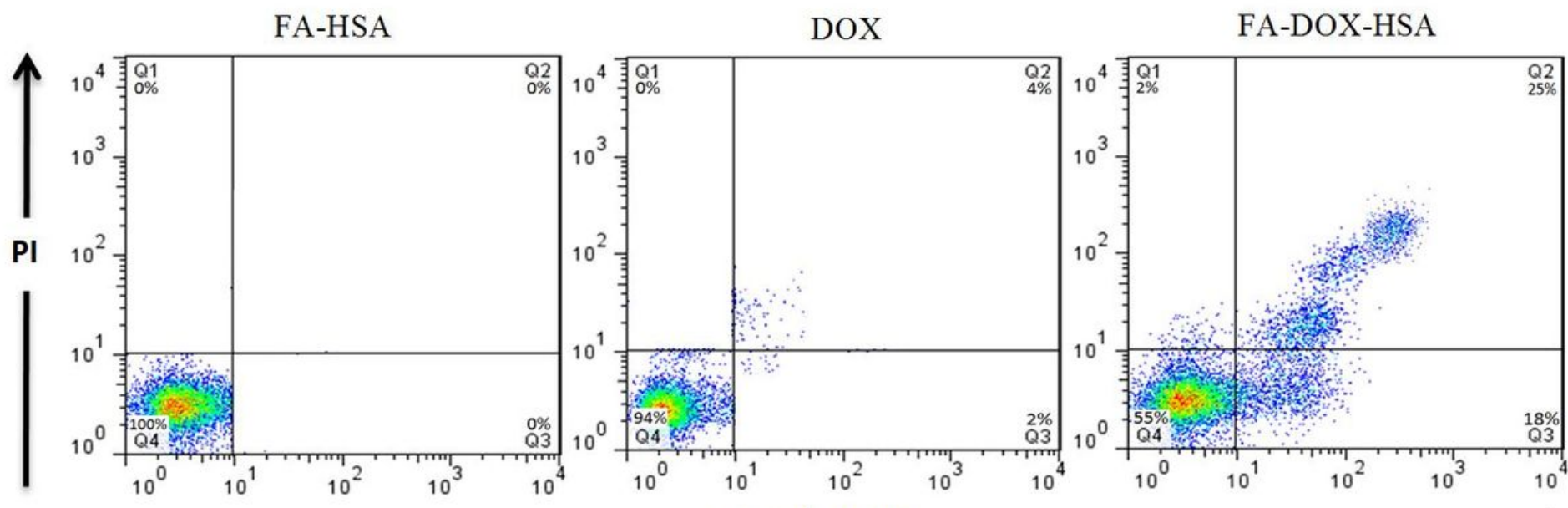

DOX

FA-DOX-HSA

Annexin V-FITC

Figure 8

Apoptosis induction by FA-DOX-HSA NPs. The RCC-GH cell line was treated with bare FA-HSA, void DOX, and FA-DOX-HSA. The number of RCC-GH cells undergoing apoptosis significantly increases when 
treating with FA-DOX-HSA NPs; Moreover, treatment of RCC-GH cell line with bare FA-HSA and void DOX separately, indicate that both treatments didn't show any remarkable apoptosis induction.

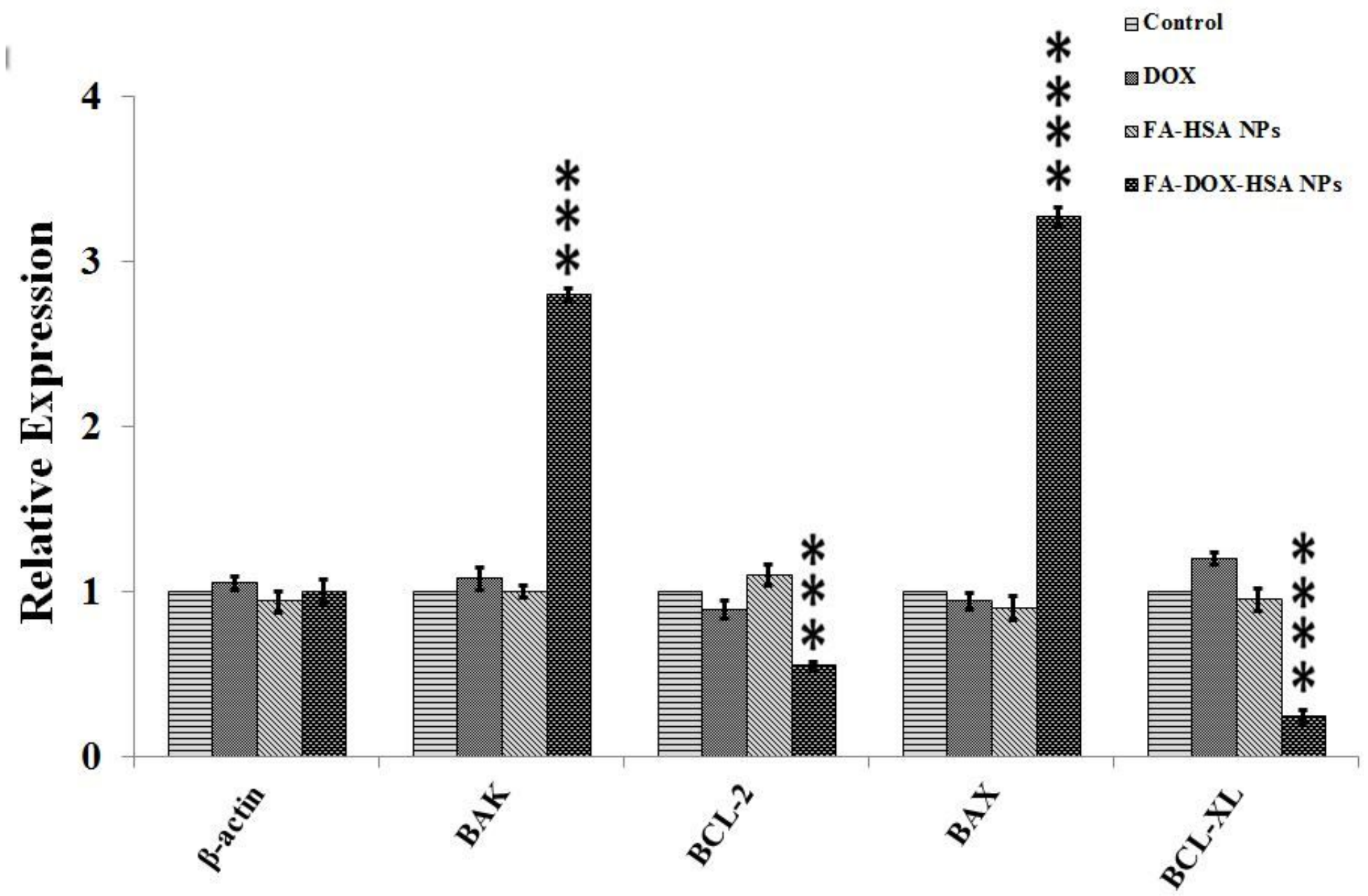

Figure 9

Real-time PCR gene expression analysis of RCC-GH cell lines treatments with void DOX, FA-HSA, and FADOX-HSA by two-way ANOVA and Bonferroni post-test. Values in the graph represent mean's ${ }^{* \star *} P<0.001$, $\star \star \star \star x<0.0001$ indicates significant differences between control (untreated) and other treatments. 